\title{
AS DISFUNÇÕES RELACIONADAS AOS TERMOS DE REFERÊNCIA DE COMPRAS PÚBLICAS E POSSÍVEIS SOLUÇÕES
}

\author{
THE DYSFUNCTIONS RELATED TO TERMS OF REFERENCE OF \\ PUBLIC PROCUREMENT AND POSSIBLE SOLUTIONS
}

Roquemar de Lima Baldam

Universidade Federal do Espírito Santo, ES, Brasil E-mail: roquemar3@gmail.com

\author{
Aline Nunes Garcia \\ Universidade Federal do Espírito Santo, ES, Brasil \\ E-mail: alinenunesgarcia@yahoo.com.br \\ Leonardo Carneiro Costa \\ Universidade Federal do Espírito, ES, Brasil \\ E-mail: leonardocarneirocosta@gmail.com \\ Erivelton Guizzardi \\ Instituto Federal do Espírito Santo, ES, Brasil \\ E-mail: eguizzardi@yahoo.com.br \\ Kayque Pereira de Paula \\ Universidade Federal do Espírito, ES, Brasil \\ E-mail:kayque.depaula3@gmail.com
}

Recebido em: 04.05.2020 - Aceito em: 25.08.2020

DOI: http://dx.doi.org/10.5902/2526629244066

RESUMO: Este estudo tem por objetivo identificar as principais falhas ou disfunções relacionadas à elaboração dos Termos de Referência (TRs) utilizados para realizar as compras públicas, bem como propor ações necessárias para eliminar ou mitigar as disfunções encontradas. Um Termo de Referência de compra pública é um documento que subsidia o setor de compras/licitações de instituições públicas com todas as informações necessárias para efetivar as aquisições de bens e serviços. A ocorrência de falhas na elaboração desse tipo de documento pode implicar atrasos na execução do processo de compra e na compra de produtos ou serviços diferentes daqueles almejados, implicando desperdícios de recursos públicos. Assim, realizou-se uma pesquisa com um grupo de foco em uma instituição pública, utilizando-se o método de análise de conteúdo para estudar os dados obtidos e estruturar os resultados. Identificaram-se duas categorias de disfunções: uma que mostra as dificuldades encontradas para elaborar TRs e outra relativa aos erros mais frequentes encontrados em TRs. Os resultados in- 
dicam a importância da implantação de comitês para realizar a análise preliminar dos projetos, com vistas à correção de erros ou falhas constantes nos TRs, antes da abertura do respectivo processo. Além disso, são apontadas possibilidades de solução em duas vertentes: desenvolvimento de um sistema de gestão da informação para elaboração de TRs; e capacitação dos servidores.

Palavras-chave: Termo de Referência; Disfunções; Compra Pública; Administração Pública.

ABSTRACT: The objective of the present study is identifying the main flaws or dysfunctions related to the formulation of Terms of Reference (TRs), used for public procurement, as well as proposing the necessary steps to eliminate or mitigate the dysfunctions found. The Term of Reference of public procurement is a document that supports the department of procurement/bidding in public institutions, with all necessary information to accomplish the purchase of goods and services. The occurrence of flaws in the formulation of this type of document may result in delays in the execution of the process of procurement or may result in the purchase of goods or services different from the ones previously expected, causing losses of public resources. Thus, one developed a research with a focus group in a public institution, using the method of content analysis to study the acquired data and to structure results. Two categories of dysfunctions were found: one of them shows the difficulties related to the formulation of TRs and the other one is related to the most frequent mistakes found in TRs. The results show the importance of the implementation of committees to conduct the preliminary analysis of projects, in order to correct the mistakes or flaws in TRs, before the beginning of the process. Besides, two possibilities for solution were indicated: development of a information management system for the formulation of TRs; and staff training.

Keywords: Term of Reference; Dysfunctions; Public Procurement; Public Administration.

\section{INTRODUÇÃO}

Muitas pesquisas procuram abordar diversas possibilidades de melhoria dos procedimentos licitatórios em contratações públicas. Estudam-se tanto as estruturas das legislações vigentes (Klun \& Setnikar-Cankar, 2013), quanto as formas de melhorar o trabalho de seleção das propostas apresentadas pelos licitantes (Simon \& Melese, 2011; Rao, Zhao \& Ma, 2012; Falagario et al., 2012), propondo alterações na fase licitatória, de forma que se possa obter melhor qualidade 
(Spagnolo, 2012) ou redução do custo total da aquisição (Costantino et al., 2012). Sabemos que, cada vez mais, as organizações vêm buscando manter-se no ambiente por meio da adoção de melhores práticas, tentando superar barreiras e otimizar facilitadores (Castro et al., 2017).

Há outra vertente de estudos que procura avaliar como as compras públicas podem ser utilizadas para promover políticas de fomento à inovação (Edquist \& Zabala-Iturriagagoitia, 2012) e à responsabilidade social corporativa (Snider et al., 2013) ou para incentivar práticas relacionadas com a sustentabilidade ambiental.

Um fato importante a ser destacado quando se trata de Administração Pública (AP) é que, em geral, independente do que se deseja comprar, o volume da compra é bastante grande. As compras públicas respondem por aproximadamente $15 \%$ dos recursos monetários movimentados no mundo (Bergman \& Lundberg, 2013). Contudo, o fornecimento de produtos e serviços para a AP não é simples, pois o processo de aquisição pública tem seus procedimentos disciplinados por regulamentações que restringem as possibilidades de contratação (Tadelis, 2012). Como aponta Moraes e Coelho (2018), a administração pública contemporânea está inserida em um contexto complexo, principalmente no que tange à fragmentação dos processos administrativos de formulação e implementação de políticas, programas e projetos.

Todavia, poucos estudos procuram propor soluções para a gestão dos processos de compras públicas, principalmente os que tratam diretamente sobre o Termo de Referência (TR), como a pesquisa realizada por Gransberg e Barton (2007).

A elaboração do termo de referência está diretamente ligada ao sucesso da contratação, uma vez que este documento subsidia o setor de compras/licitações com todas as informações necessárias para efetivar determinada aquisição, servindo de base para realizar o procedimento licitatório. Um TR construído de forma inadequada pode acarretar prejuízo para a AP, seja pelo atraso na execução do processo de compra, seja pela compra de produto com especificações diferentes das almejadas ou em quantidade insuficiente.

Em face do exposto, torna-se necessário pesquisar como melhorar a elaboração de TRs para os processos de contratação pública, atentando para as especificações e condições necessárias para atender aos anseios do setor que elabora a demanda pelo bem ou serviço a ser adquirido.

Neste contexto, a pesquisa realizada teve como objetivo identificar as principais disfunções relacionadas à elaboração dos TRs em processos de aquisições públicas, assim como indicar possíveis ações para eliminar ou mitigar as disfun- 
ções encontradas, contribuindo para otimizar o processo de compra e, consequentemente, o uso dos recursos públicos.

\section{REFERENCIAL TEÓRICO}

Nesta seção, procura-se expor os estudos atuais que se relacionam com a elaboração e o uso do TR para compras públicas, abordando a sua aplicabilidade, seu uso relacionado com compras conjuntas, bem como o contexto da gestão de processos de negócios no qual ocorre a sua utilização.

\subsection{A Aplicação do Termo de Referência}

Uma importante função do TR é definir os critérios para a seleção das propostas apresentadas em um processo de compra pública. Entre os estudos recentes que se relacionam com o TR, destaca-se o realizado por Xia et al. (2013), que ao analisarem os critérios de seleção adotados em TR, constataram que, embora o preço seja a categoria mais importante de seleção, a sua importância relativa diminuiu significativamente na última década. As categorias de qualificação, experiência e desempenho anteriores tornaram-se mais importantes para os contratantes.

Um exemplo desta função pode ser percebido no estudo de Azambuja e O'Brien (2012), ao mostrarem que a aquisição de equipamentos é um processo complexo, que requer a avaliação de vários fornecedores. A análise geralmente é realizada de forma manual, sendo demorada e, assim, certas vantagens e desvantagens podem ser negligenciadas. Neste contexto, os autores propuseram o uso de um software de apoio à seleção de fornecedores que se destaca por permitir sua interação com o usuário, apresentar linguagem de fácil compreensão e possibilitar o rápido cadastramento e atualização do banco de dados.

Vale ressaltar que a aplicação de softwares tem sido cada vez mais utilizada na gestão pública para fornecimento de informações. Exemplo disso é sua aplicação no processamento de pedidos (Chen et al., 2013) e consultas (Choi \& Chung, 2013), podendo ser utilizada inclusive para interpretação de indicadores de desempenho (Tomic \& Milic, 2013). Contudo, observa-se que para avaliar as propostas em um processo de aquisição pública, seja manualmente ou por meio do uso de software, o TR precisa estabelecer de forma clara e precisa quais critérios de seleção devem ser adotados. O uso de software não resultará em uma boa contratação, caso o TR apresente deficiências no estabelecimento desses critérios.

O TR tem por objetivo, também, definir precisamente o objeto e o seu custo 
de contratação, bem como as condições para o fornecimento dos bens ou para a realização dos serviços, estabelecendo as obrigações do fornecedor contratado e da instituição contratante (Brasil, 2000).

\subsection{Termos de Referência em Compras Compartilhadas}

A fim de propiciar maior agilidade ao processo de compra, otimizando principalmente os esforços, alguns estudiosos apostam na compra conjunta. Trata-se de um tipo de aquisição em que há colaboração entre várias organizações, as quais agrupam e compartilham volumes, informações e recursos de compras (Schotanus et al., 2011). Para Walker et al. (2013), os governos estimulam ou forçam a utilização das compras públicas compartilhadas como forma de reduzir as ineficiências do sistema de aquisições. Conforme Huff-Rousselle (2012), com a realização de compras compartilhadas, a administração pública consegue obter redução dos preços, melhoria da qualidade do que é adquirido, além da redução de custos operacionais e administrativos.

Contudo, como qualquer tipo de aquisição, a compra conjunta apresenta aspectos positivos e negativos (Huff-Rousselle, 2012). Alguns deles estão listados no Quadro 1.

Quadro 1 - Destaques positivos e negativos da aquisição conjunta, adaptado de HuffRousselle (2012).

\begin{tabular}{|c|c|}
\hline Destaques Positivos & Destaques Negativos \\
\hline Redução do preço unitário & Decisões políticas \\
\hline Economia de escala & $\begin{array}{l}\text { Leis ou regulamentações locais ou } \\
\text { procedimentos operacionais }\end{array}$ \\
\hline Poder de negociação & "Lobby" de fornecedores \\
\hline $\begin{array}{l}\text { Melhoria de qualidade por meio de } \\
\text { medidas preventivas }\end{array}$ & $\begin{array}{l}\text { Se houver pagamento atrasado a } \\
\text { fornecedores, pode-se enfraquecer o poder } \\
\text { de compra }\end{array}$ \\
\hline $\begin{array}{c}\text { Redução ou eliminação da corrupção nos } \\
\text { contratos }\end{array}$ & $\begin{array}{l}\text { Participação de fornecedores de } \\
\text { desempenho questionável }\end{array}$ \\
\hline $\begin{array}{l}\text { Redução de custos operacionais e } \\
\text { encargos administrativos }\end{array}$ & Falta de padronização \\
\hline
\end{tabular}

Fonte: Adaptado de Huff-Rousselle (2012) 
Para realizar compras compartilhadas, a administração pública no Brasil utiliza um modelo de contratação denominado Sistema de Registro de Preços SRP. De acordo com a legislação brasileira, é indicado utilizar o registro de preços quando: a administração não sabe ao certo a quantidade que será consumida do produto durante determinado período; há necessidade de contratações frequentes do bem ou serviço; for conveniente a aquisição com previsão de entregas parceladas; for conveniente adquirir para mais de um órgão ou entidade (Brasil, 2013).

Na compra realizada por um SRP há uma instituição gerenciadora da aquisição e outras participantes que solicitam a inclusão de itens e quantitativos para atender às suas necessidades. Nesse tipo de operação, deficiências no TR têm um impacto muito maior, uma vez que vários órgãos poderão participar do mesmo processo de compras, e os prejuízos que serão trazidos para o órgão gerenciador se estenderão aos demais participantes do SRP.

Em um SRP, uma das funções de um TR é realizar uma estimativa das quantidades a serem adquiridas. Porém, a administração pode decidir pela não contratação caso conclua que esta não é necessária. Em face do exposto, sendo a única garantia do fornecedor a preferência na contratação, caso ele tenha o meIhor preço na época da referida contratação, o mesmo tende a esperar um lucro menor (Xu, Chen \& Xu, 2010). Por consequência, para fins de registro de preços, o fornecedor estabelece um preço maior do produto de modo a compensar a incerteza quanto à contratação. Assim, o ganho de escala do contratante pode ficar comprometido com a compra compartilhada realizada pelo SRP.

Nos processos de compra, deve-se considerar a incerteza relacionada à contratação (Darwish, Abdulmalek \& Alkhedher, 2013; Zhang \& Chen, 2013), e também a existência de incertezas relacionadas ao fornecimento (Xu \& Lu, 2013).

Ao pesquisarem sobre a incerteza de fornecimento, visto que a quantidade recebida pode ser diferente da quantidade solicitada, Xu e Lu (2013) concluíram que o efeito da incerteza de abastecimento, apesar de não ser universal, afeta o rendimento e, consequentemente, o preço de venda.

Quanto à incerteza da demanda, vale destacar a pesquisa sobre a seleção de fornecedores e os problemas da decisão de aquisição em função da demanda incerta, descontos em função da quantidade (economia de escala) e custos de seleção fixos, desenvolvida por Zhang e Chen (2013). De acordo com a referida pesquisa, a escolha do fornecedor não é uma decisão discricionária e quando se trata de instituições públicas, apenas em alguns casos ela é possível. Entretanto, os problemas de decisão de aquisição em função da demanda incerta e des- 
contos em função da quantidade é uma regra geral quando se trata de um SRP. Obviamente, quando há discrepâncias no TR, os descontos são menores e, portanto, não será feita a aquisição mais vantajosa para a administração, conforme determina a lei de licitações e contratos brasileira.

\subsection{A Gestão de Processos de Negócios na Administração Pública}

Segundo Pyon, Woo e Park (2011), a melhoria da gestão de processos é de suma importância para a melhoria da qualidade do serviço, devendo-se considerar tanto os dados mensuráveis quanto os não mensuráveis, tais como: indicadores de desempenho e feedback dos clientes. Esse desempenho é positivamente influenciado pela implementação da gestão da qualidade (Pereira-Moliner et al., 2012). Uma abordagem holística consagrada para a gestão e melhoria dos processos organizacionais, cujas raízes estão na Gestão da Qualidade Total (TQM) e na Reengenharia de Processos de Negócios (BPR), é a Gestão de Processos de Negócios (Business Process Management - BPM). Muitas pesquisas têm procurado entender como as organizações podem desenvolver suas capabilidades de BPM, que são as habilidades de uma organização para integrar, construir e reconfigurar seus processos, visando adequar-se ao ambiente de mercado (Niehaves, Plattfaut \& Becker, 2013). Nesse sentido, ao pesquisarem sobre as capabilidades de BPM em governos locais, declaram que é notória a percepção de que as organizações do setor público precisam reavaliar seus processos de negócios. Gomes e Machado (2018) pontuaram que um grande desafio enfrentado pelo setor público consiste em fornecer serviços de qualidade com recursos escassos e capacidade operacional limitada.

O BPM também pode ser entendido como uma melhoria contínua de processos, a qual possui fatores críticos que, ao serem melhorados, permitem que as organizações desenvolvam uma orientação voltada para processos de negócios (Skrinjar \& Trkman, 2013). Em um sentido mais prático, o BPM integra um conjunto de tecnologias capazes de traduzir os modelos de processos de negócios em atividades computacionais com suporte (Antunes \& Mourão, 2011).

A gestão dos processos de uma organização passa pela modelagem dos mesmos. Turetken e Demirors (2011) defendem que a modelagem dos processos seja feita de forma descentralizada, o que proporciona várias vantagens, entre as quais: maior grau de envolvimento entre as pessoas; redução no tempo gasto para modelagem; atividades diárias para quem modela; e participação, motivação e compromisso de todos os envolvidos. Desempenho, comprometimento organi- 
zacional, comprometimento organizacional afetivo e busca de desenvolvimento de habilidades apresentaram correlação positiva com o construto comprometimento com a carreira (Santos, 2017).

Dessa forma, vários estudos mais recentes procuram avaliar a melhoria da gestão da qualidade em setores públicos e privados (Oliveira, Serra \& Salgado, 2010; Chen \& Chen, 2011; Yu, To \& Lee, 2012; Kim, Kumar \& Kumar, 2012; Asif \& Raouf, 2013).

Yu, To e Lee (2012) validaram o modelo de Sistema de Gestão de Qualidade baseado em processos para as organizações públicas, reforçando a ligação com os princípios de qualidade. No estudo, os autores ressaltaram a importância dos fatores humanos e do investimento de recursos em pessoal, por parte das organizações públicas, além de treinamento de seus líderes. Kim, Kumar e Kumar (2012) consideram a gestão de qualidade como uma filosofia de gestão holística que favorece todas as funções de uma organização por meio de melhoria contínua e mudança organizacional. Defendem que este tipo de gestão gera satisfação do cliente, inovação e melhor desempenho da empresa.

Tazelaar e Snijders (2013) citam o seguinte paradoxo: embora profissionais mais experientes tendam a decidir de formas diferentes, muitas vezes eles não fazem melhor avaliação do que aqueles com menos experiência. Os resultados obtidos pelo estudo dos autores mostram que o conhecimento contribui com a maior certeza sobre as avaliações e a experiência contribui com a maior utilização do julgamento intuitivo. Daí o entendimento de que para a elaboração de TR a experiência é importante, em virtude principalmente das informações que se deve prestar em sua elaboração. Considerando tal fato, os autores concluem que a vantagem que detém os mais experientes poderia ser diminuída ou chegar a zero, caso houvesse alguma ferramenta tecnológica que auxiliasse servidores novatos a elaborarem o referido documento.

Ramakrishnan, Jones e Sidorova (2012) investigaram a existência de pressões externas e internas à organização, que a levariam a implementar um Business Inteligence $(B L)$. Os resultados nos ajudam a entender melhor os propósitos do $\mathrm{BI}$ e indicam fatores de sucesso relacionados à coleta de dados. A pesquisa verificou que as escolhas estratégicas e táticas não são influenciadas por pressões externas como competição, mas sofrem influência de pressões internas da organização. Portanto, no caso de se pleitear a adoção de ferramentas de BI para a elaboração de TR, a pressão para a utilização desse tipo de recurso poderá vir da própria instituição diante dos fracassos nas aquisições anteriores. 
El-Haddadeh, Weerakkody e Al-Shafi (2013) afirmam que a implementação de serviços eletrônicos no setor público procura melhorar a eficiência, a eficácia e a transparência dos departamentos governamentais. Ao analisarem a complexidade desta implementação, os autores elencaram como principais problemas: a falta de prontidão e consciência; a falta de confiança; as preocupações com privacidade e segurança; a resistência à mudança; a falta de habilidades; e a falta de apoio do próprio governo. Segundo os autores, a exclusão digital e a falta de acesso ao sistema influenciam na confiabilidade e, consequentemente, impedem a inclusão de novos serviços eletrônicos.

Nesse contexto, considerando que na Administração Pública fala-se basicamente em serviços, ressalta-se a revisão da literatura realizada por Lane e Richardson (2011), a qual documenta o estado da arte em desenvolvimento de modelos de processos para aplicações em serviços. Portanto, este estudo pode ser considerado uma importante fonte de informação para o entendimento dos modelos de compra existentes e da melhoria da fase de elaboração de TRs.

\section{METODOLOGIA DA PESQUISA}

Haja vista o objetivo do presente estudo, por meio do qual se pretende identificar as disfunções existentes na elaboração dos TRs de processos de compras públicas, bem como propor possíveis soluções, optou-se por realizar uma pesquisa com um grupo de foco, de forma a captar, na prática, a percepção dos principais atores envolvidos nos processos de contratação, inclusive na elaboração de Termos de Referência. A pesquisa foi aplicada em uma instituição pública que será identificada somente por IP. Sua identidade será omitida a pedido de seu dirigente.

A metodologia adotada pode ser dividida em duas fases: i) planejamento e recrutamento dos participantes e ii) implementação da sessão com o grupo de foco. A seguir, é apresentado o breve detalhamento dessas fases nos itens 3.1 e 3.2.

\subsection{Planejamento do grupo e recrutamento dos participantes}

Sendo o grupo de foco um método em que se reúne um grupo de oito a doze pessoas para discutir um assunto sob a coordenação de um moderador (Rea \& Parker, 2002), decidiu-se realizar a pesquisa com um grupo de doze pessoas envolvidas diretamente em processos de compra, desde a etapa de elaboração da requisição até a efetiva aquisição. Para a discussão em grupo, foi proposto o tema "disfunções mais frequentes em TRs e possíveis soluções em face do(s) respectivo(s) problema(s)". 
A instituição pública escolhida realiza um volume considerável de compras, seguindo os preceitos da legislação brasileira. A atividade principal desta IP é prestar serviços a proprietários, condutores e usuários de veículos e transportes terrestres, aplicar penalidades e sanções, emitir documentos, licenças e autorizações a esses usuários, entre outras atividades. Entretanto, as aquisições realizadas pelo órgão não se limitam à área de atuação da IP. Apesar de existirem várias aquisições bem específicas (por exemplo, tinta para sinalização viária), muitas contratações da IP são de materiais e serviços comuns, isto é, necessários em quaisquer instituições públicas, independentemente de sua área de atuação (manutenção de condicionadores de ar e elevadores, aquisição de livros, de materiais gráficos, produtos de limpeza, materiais de escritório, etc.). Pelo fato da IP estar presente em todos os municípios do Estado onde está localizada, e serem vários os setores demandantes por compras públicas, existe uma variabilidade grande de objetos a serem adquiridos e, consequentemente, a quantidade e variabilidade de disfunções em TRs também são consideráveis, o que torna o ambiente organizacional dessa instituição um campo propício para a realização da presente pesquisa.

Com o objetivo de reunir o conhecimento dos diferentes servidores (altamente experientes ou menos experientes) envolvidos no processo de contratação, foram convidados a participar do grupo de discussão: oito pessoas que detêm grande conhecimento prático e teórico no que diz respeito ao processo de contratação e quatro pessoas que já tiveram que elaborar TR, mas que não detinham muitas informações sobre o referido documento, além de não terem sido treinadas para tal atividade. A decisão de convocar pessoas com menor conhecimento sobre o assunto justifica-se pela necessidade de entender as dificuldades que os servidores têm para elaborar TR, procurando obter sua opinião sobre como poderia ser melhorado o processo de elaboração do documento. Como premissa para realizar o convite aos possíveis participantes, considerou-se o interesse das pessoas em discutir o assunto, em concordância com Simon (1999), Krueger e Casey (2000) e Rea e Parker (2002).

\subsection{Implementação da sessão com o grupo de foco}

Para a pesquisa com o grupo de foco na IP, realizou-se uma sessão de discussão em formato semiestruturado, possibilitando direcionar a conversa para o assunto principal (Simon, 1999; Krueger \& Casey, 2000; Rea \& Parker, 2002), além de dar oportunidade para os participantes exporem suas ideias, comentários e soluções inovadoras sem, contudo, fugir do assunto. 
Para o desenvolvimento do estudo, foram adotadas perguntas-chave, visando direcionar uma discussão que contribuísse com os objetivos da pesquisa, evitando o desvio da atenção dos participantes para outros temas. Assim, foram consideradas as seguintes questões referenciais:

I. Quais são as principais dificuldades encontradas pelos servidores dentro do órgão para a elaboração de TRs?

II. Quais são os erros mais frequentes encontrados dentro desses documentos ao longo do processo de compra?

III. Quais seriam as soluções mais adequadas para a resolução/mitigação das disfunções na elaboração do TR?

IV. A implantação de soluções de Gestão de Processos de Negócios poderia ajudar na solução dos problemas?

V. Acreditam ser possível obter solução tecnológica para o problema? Caso afirmativo, que tipo de solução seria essa?

\subsection{Análise dos dados}

Após a realização da sessão com o grupo de foco, as informações obtidas foram organizadas e analisadas por meio do método de análise de conteúdo (Bardin, 2004), o que permitiu a seguinte estruturação dos dados:

i. Codificação das respostas - como o tema foi a unidade escolhida, procurou-se organizar as opiniões em ideias, representadas por frases, sobre as disfunções em TRs e proposições de soluções;

ii. Categorização - utilizada para agrupar as disfunções e proposições em forma de títulos genéricos, facilitando a apresentação dos resultados; e

iii. Regras de contagem - optou-se pela frequência, considerando que a importância de determinada unidade aumenta com a frequência de sua aparição.

Os resultados encontrados foram estruturados em tabelas, de modo a apresentar as disfunções relacionadas aos TRs, assim como as possíveis ações sugeridas para eliminar ou mitigar tais disfunções. Em seguida, esses resultados foram analisados pelos pesquisadores com vistas a subsidiar a proposição de sugestões de melhorias.

\section{RESULTADOS E DISCUSSÃO}

Os dados obtidos a partir da sessão com o grupo de foco, analisados e estruturados de acordo com o método de análise de conteúdo, são discutidos nesta seção e apresentados nas Tabelas 1 e 2. 
$\mathrm{Na}$ Tabela 1, são apresentadas as disfunções encontradas em termos de referência, subdivididas em duas categorias: as dificuldades encontradas pelos servidores públicos na elaboração de um TR e os erros encontrados por aqueles que avaliam o documento.

Tabela 1 - As disfunções em termos de referência para compras públicas.

\begin{tabular}{|c|c|c|}
\hline Categorias & Disfunções & Frequência \\
\hline \multirow{8}{*}{$\begin{array}{l}\text { Dificuldades para } \\
\text { elaborar TRs }\end{array}$} & $\begin{array}{c}\text { Ausência de informações quanto ao grau } \\
\text { necessário de especificação do item a ser } \\
\text { adquirido }\end{array}$ & 8 \\
\hline & $\begin{array}{l}\text { Falta de experiência e conhecimento técnico } \\
\text { para a elaboração do TR }\end{array}$ & 6 \\
\hline & Falta de treinamento e capacitação & 6 \\
\hline & Alta rotatividade de pessoal & 5 \\
\hline & $\begin{array}{c}\text { Quem elabora o TR não sabe como funciona } \\
\text { a administração pública }\end{array}$ & 3 \\
\hline & Falta de orientação sobre como montar um TR & 2 \\
\hline & $\begin{array}{c}\text { Falta de padronização dos itens a serem } \\
\text { adquiridos }\end{array}$ & 2 \\
\hline & Questões políticas internas da organização & 2 \\
\hline \multirow{12}{*}{$\begin{array}{l}\text { Erros frequentes } \\
\text { encontrados em TRs }\end{array}$} & Especificação técnica dos itens insuficiente & 11 \\
\hline & Conteúdo em desacordo com a legislação & 5 \\
\hline & Erros no emprego da língua portuguesa & 5 \\
\hline & Justificativa de interesse público inadequada & 5 \\
\hline & $\begin{array}{l}\text { Falta de informação sobre a possibilidade de } \\
\text { prorrogação do contrato }\end{array}$ & 4 \\
\hline & Má instrução do processo & 3 \\
\hline & $\begin{array}{l}\text { Falta das cláusulas "obrigações da contratada } \\
\text { e da contratante" }\end{array}$ & 3 \\
\hline & Falta da cláusula de "fiscalização do contrato" & 2 \\
\hline & Falta cláusula "forma de entrega" & 2 \\
\hline & Inadequada organização do documento & 2 \\
\hline & $\begin{array}{c}\text { Ausência de declaração sobre "bem ou } \\
\text { serviço comum" }\end{array}$ & 2 \\
\hline & Cláusulas repetitivas & 1 \\
\hline
\end{tabular}

Fonte: Elaborada pelos autores a partir da análise de conteúdo dos dados obtidos com o grupo de foco

A partir da análise dos dados obtidos, percebe-se que os participantes fizeram sugestões e críticas diretamente ligadas às suas áreas de atuação. As pessoas que trabalham no setor de compras, por exemplo, destacaram disfunções relacionadas principalmente às especificações dos produtos ou serviços e à desorganização do documento, as quais afetam diretamente seus trabalhos diários. Entre as principais dificuldades encontradas pelos servidores destacam-se: a au- 
sência de informações quanto ao grau necessário de especificação do item a ser adquirido (8), a falta de experiência e de conhecimento técnico para a elaboração do TR (6), a falta de treinamento e capacitação (6) e a alta rotatividade de pessoal (5), sendo o erro mais comum correspondente à especificação técnica dos itens insuficiente (11). Além disso, foram apontados erros de conteúdo em desacordo com a legislação (5) e no emprego da língua portuguesa (5), além da inadequação da justificativa de interesse público para a contratação (5).

Também chama a atenção o relato quanto à ocorrência de erros no emprego da língua portuguesa constantemente presentes em documentos; não apenas em TRs, mas em diversos outros documentos elaborados por servidores da instituição. Esse fato é preocupante na medida em que leva à reflexão mais ampla acerca do perfil de alguns profissionais que trabalham nas instituições públicas. Além disso, ainda há a alta rotatividade dentro dos órgãos públicos, o que não contribui para a eficiência da instituição, já que quando o profissional passa a entender de fato o sistema a ponto de contribuir de forma mais efetiva, a direção recebe o pedido de desligamento/exoneração. Esse tipo de resultado nos leva a pensar sobre quais são os critérios utilizados para a nomeação de pessoas em cargos comissionados (de livre nomeação e livre exoneração) pelos gestores. Será que estão sendo nomeados servidores sem instrução suficiente para exercer cargos e funções públicas? Será a nomeação de servidores mero favor político? Estes são alguns questionamentos importantes, que apesar de não serem alvos deste estudo, mereceriam estudos específicos com vistas à obtenção de respostas fundamentadas.

Tabela 2 - Propostas de ações para eliminar ou mitigar as disfunções em termos de referência.

\begin{tabular}{ccc}
\hline Categorias & Soluções propostas & Frequência \\
\hline $\begin{array}{c}\text { Ações mais } \\
\text { adequadas para } \\
\text { a resolução } \\
\text { ou mitigação } \\
\text { dos problemas } \\
\text { (disfunções) }\end{array}$ & Capacitação específica & 8 \\
\hline & $\begin{array}{c}\text { Triagem preliminar dos projetos antes da } \\
\text { autuação dos processos }\end{array}$ & 7 \\
\hline Capacitação geral \\
Capacitação permanente dentro do próprio \\
órgão
\end{tabular}




\begin{tabular}{lcc}
\hline & Melhorias na comunicação interna & 2 \\
\hline & $\begin{array}{c}\text { Treinamento para chefes e fiscais de contratos } \\
\text { Estabelecimento de diretrizes para a elaboração } \\
\text { de TR }\end{array}$ & 2 \\
\hline & Trabalhar em rede / cultura da cooperação & 1 \\
\hline $\begin{array}{c}\text { Soluções } \\
\text { tecnológicas para o } \\
\text { problema }\end{array}$ & Tutorial para elaboração de TRs & 1 \\
\hline & $\begin{array}{c}\text { Sistema para organizar pedidos de compra } \\
\text { (levantamento de demanda antes da compra) }\end{array}$ & 5 \\
\hline $\begin{array}{c}\text { Banco de dados com legislação aplicável ao } \\
\text { processo de compras. }\end{array}$ & 2 \\
\hline $\begin{array}{l}\text { Fonte: Elaborada pelos autores a partir da análise de conteúdo dos dados obtidos com o } \\
\text { grupo de foco }\end{array}$ & 5
\end{tabular}

A respeito das possíveis soluções para os problemas em TRs, conforme Tabela 2, a maioria dos participantes apontou a necessidade de capacitação específica (8) sobre o tema, sendo recomendada a capacitação contínua em função da constante rotatividade de servidores na IP. Este tipo de proposta se relaciona com as necessidades de investimento de recursos em pessoal e de treinamento dos líderes em organizações públicas, identificadas por Yu, To e Lee (2012).

A segunda proposta mais comum entre os participantes foi a realização de uma triagem preliminar dos projetos antes da autuação dos processos, visando a correção de erros no documento (7). Acerca desta solução, foi informado pelos participantes do grupo de foco que está em fase de implantação na IP um Comitê de Avaliação de Projetos e Processos. Segundo relatos do dirigente e de servidores da IP, apesar de haver resistência por parte de alguns servidores da instituição, em função da demora em autuar o processo, já é possível perceber uma melhora considerável na sua execução. Um dos participantes do grupo de foco relatou que "os atrasos que ocorriam em função dos erros no TR tem diminuído consideravelmente".

Quando questionados sobre possíveis soluções tecnológicas, a ampla maioria entendeu ser possível obter solução tecnológica para eliminar ou mitigar as disfunções relacionadas aos termos de referência em processos de contratação do setor público; seis dos doze participantes pensaram na utilização de um banco de dados contendo especificações de produtos e materiais, além da disponibilização de TRs prontos que servissem de modelo à elaboração de outros TRs. Outra solução tecnológica levantada foi o desenvolvimento de um tutorial para elaboração de TRs. Este tipo de solução está em consonância com o uso de softwares na gestão pública, proposto por Chen et al. (2013) e Choi e Chung (2013). 


\section{CONSIDERAÇOES FINAIS}

A partir do presente estudo, conclui-se que as principais disfunções relacionadas aos termos de referência em processos de compras públicas são as seguintes: a especificação técnica dos itens insuficiente; a ausência de informações quanto ao grau necessário de especificação do item a ser adquirido; a falta de experiência e de conhecimento técnico para a elaboração do TR; a falta de treinamento e capacitação; a alta rotatividade das pessoas dentro dos órgãos públicos; o conteúdo do TR elaborado em desacordo com a legislação vigente e com as normas da língua portuguesa; e a inadequação da justificativa de interesse público para a contratação. Além disso, foi possível perceber que algumas disfunções estão inter-relacionadas, tais como a falta de conhecimento técnico, a falta de treinamento e a alta rotatividade de pessoal.

Quanto às possíveis soluções para as disfunções relacionadas aos TRs em processos de contratação do setor público, destacam-se: a capacitação específica dos servidores, observando a área de atuação de cada servidor; a análise preliminar dos projetos, com vistas à correção de erros constantes nos TRs, antes da autuação do respectivo processo; a capacitação geral e permanente dos servidores sobre compras públicas, em função da constante rotatividade de servidores; a padronização dos TRs; e as soluções tecnológicas (como exemplo: a criação de um banco de dados com especificações de produtos e serviços; a elaboração de modelos de TRs; e tutorial para elaboração de TRs). Diante disso, o desenvolvimento de um sistema de gestão da informação e do conhecimento, além de atender às aspirações dos principais atores envolvidos nos processos de contratação do setor público, mostra-se uma importante alternativa para a solução das disfunções identificadas por este estudo nos processos de compras públicas.

Assim, a fim de tornar a administração pública mais ágil e eficiente, otimizando a utilização dos recursos públicos, recomendamos estudos específicos voltados à implementação de soluções tecnológicas, visando obter maior eficiência nos processos de compra, bem como investimentos internos voltados à efetiva implantação dos comitês de avaliação de projetos e processos, haja vista que o comitê da instituição pesquisada tem mostrado importantes resultados, especialmente na elaboração de TRs, contribuindo para a melhoria das aquisições públicas. 


\section{REFERENCIAS}

Antunes, P., \& Mourão, H. (2011). Resilient business process management: framework and services. Expert Systems with Applications, 38, 1241-1254.

Asif, M., \& Raouf, A. (2013). Setting the course for quality assurance in higher education. Quality and Quantity, 47, 2009-2024.

Azambuja, M. M., \& O'Brien, W. J. (2012). Rapid assessment and selection of engineered equipment suppliers. Automation in Construction, 22, 587-596.

Bardin, L. (2004). Análise de Conteúdo. (3. ed). Lisboa: Edições 70.

Bergman, M. A., \& Lundberg, S. (2013). Tender evaluation and supplier selection methods in public procurement. Journal of Purchasing and Supply Management, 19(2), 73-83.

Brasil. (2000). Decreto $\mathrm{n}^{\circ} 3.555$, de 08 de agosto de 2000. Aprova o regulamento para a modalidade de licitação denominada pregão, para aquisição de bens e serviços comuns. Recuperado de http://www.planalto.gov.br/ccivil_03/decreto/D3555.htm.

. (2013). Decreto $\mathrm{n}^{\circ}$ 7.892, de 23 de janeiro de 2013. Regulamenta o Sistema de Registro de Preços previsto no art. 15 da Lei $n^{\circ}$ 8.666, de 21 de junho de 1993. Recuperado de http://www.planalto.gov.br/ccivil_03/_Ato20112014/2013/Decreto/D7892.htm.

Chen, J., \& Chen, I. S. (2011). Inno-Qual efficiency of higher education: empirical testing using data envelopment analysis. Expert Systems with Applications, 38, 1823-1834.

Chen, J., Lee, V. C. S., Liu, K., Ali, G. G. M. N., \& Chan, E. (2013). Efficient processing of requests with network coding in on-demand data broadcast environments. Information Sciences, v. 232, p. 27-43.

Choi, D., \& Chung, C. (2013). Request: a framework for efficient processing of region-based queries in sensor networks. Information Sciences, 248, 151-167.

Costantino, N., Dotoli, M., Falagario, M., \& Sciancalepore, F. (2012). Balancing the additional costs of purchasing and the vendor set dimension to reduce public procurement costs. Journal of Purchasing \& Supply Management, 18, 189-198.

De Castro, C., Isidro-Filho, A., Menelau, S., \& Fernandes, A. (2017). Antecedentes de inovações em organizações públicas do poder executivo federal. CadernosGestão Pública E Cidadania, 22(71).

Darwish, M. A., Abdulmalek, F., \& Alkhedher, M. (2013). Optimal selection of process mean for a stochastic inventory model. European Journal of Operatiomean for a stochastic inventory model. European Journal of Operational Research, 226, 481-490. 
Edquist, C., \& Zabala-Iturriagagoitia, J. M. (2012). Public procurement for innovation as mission-oriented innovation policy. Research Policy, 41, 1757-1769.

El-Haddadeh, R., Weerakkody, V., \& Al-Shafi, S. (2013). The complexities of electronic services implementation and institutionalization in the public sector. Information \& Management, 50,135-143.

Falagario, M., Sciancalepore, F., Costantino, N., \& Pietroforte, R. (2012). Using a DEA-cross efficiency approach in public procurement tenders. European Journal of Operational Research, 218, 523-529.

Gransberg, D. D., \& Barton, R. F. (2007). Analysis of federal design-build request for proposal evaluation criteria. Journal of Management in Engineering, 23(2), 105-111.

Gomes, C., \& Machado, A. (2018). Fatores que influenciam a inovação nos serviços públicos: o caso da Secretaria Municipal de Saúde de Campina Grande. Cadernos Gestão Pública E Cidadania, 23(74).

Huff-Rousselle, M. (2012). The logical underpinnings and benefits of pooled pharmaceutical procurement: a pragmatic role our public institutions? Social Science \& Medicine, 75, 572-1580.

Kim, D., Kumar, V., \& Kumar, U. (2012). Relationship between quality management practices and innovation. Journal of operations management, 30, 295-315.

Klun, M., \& Setnikar-Cankar, S. (2013). Better regulation and public procurement in Slovenian municipalities. Transylvanian Review of Administrative Sciences, 88, 96-105.

Krueger, R., \& Casey, M. A. (2000). Focus Groups: a practical guide for applied research. (3. ed). London: Sage.

Lane, S., \& Richardson, I. (2011). Process models for service-based applications: a systematic literature review. Information and Software Technology, 53, 424-439.

Moraes, L., \& Coelho, F. (2018). (Des)integração entre os subsistemas de ingresso no serviço público: Análise de uma carreira do ciclo de gestão. Cadernos Gestão Pública E Cidadania, 23(75).

Niehaves, B., Plattfaut, R., \& Becker, J. (2013). Business process management capabilities in local governments: a multi-method study. Government Information Quarterly, 30, 217-225.

Oliveira, O. J. de, Serra, J. R., \& Salgado, M. H. (2010). Does ISO 14001 work in Brazil? Journal of Cleaner Production, 18, 1797-1806. 
Pereira-Moliner, J., Claver-Cortés, E., Molina-Azorín, J. F., \& Tarí, J. J. (2012). Quality management, environmental management and firm performance: direct and mediating effects in the hotel industry. Journal of Cleaner Production, 37, 82-92.

Pyon, C. U., Woo, J. Y., \& Park, S. C. (2011). Service improvement by business process management using customer complaints in financial service industry. Expert Systems with Applications, 38, 3267-3279.

Ramakrishnan, T., Jones, M. C., \& Sidorova, A. (2012). Factors influencing business intelligence $(\mathrm{BI})$ data collection strategies: an empirical investigation. Decision Support Systems, 52, 486-496.

Rao, C., Zhao, Y., \& Ma, S. (2012). Procurement decision making mechanism of divisible goods based on multi-attribute auction. Electronic Commerce Research and Applications, 11, 397-406.

Rea, L. M., \& Parker, R. A. (2002). Metodologia de pesquisa: do planejamento à execução. São Paulo: Pioneira Thomson Learning.

Santos, A. (2017). Comprometimento e entrincheiramento em carreiras do setor público: estudo exploratório e confirmatório. Cadernos Gestão Pública E Cidadania, 22(73).

Schotanus, F., Walker, H., Bakker, E., \& Essig, M. (2011). Development of purchasing groups during their life cycle: from infancy to maturity. Public Administration Review, 71, 265-275.

Simon, J., \& Melese, F. (2011). A multiattribute sealed-bid procurement auction with multiple budgets for government vendor selection. Decision Analysis, 8 , 170-179.

Simon, J. S. (1999). Conducting Successful Focus Groups. Saint Paul: Wilder Publishing Center.

Skrinjar, R., \& Trkman, P. (2013). Increasing process orientation with business process management: critical practices. International Journal of Information Management, 33, 48-60.

Snider, K. F., Halpern, B. H., Rendon, R. G., \& Kidalov, M. V. (2013). Corporate social responsibility and public procurement: how supplying government affects managerial orientations. Journal of Purchasing \& Supply Management, $19,63-72$.

Spagnolo, G. (2012). Reputation, competition, and entry in procurement. International Journal of Industrial Organization, 30, 291-296.

Tadelis, S. (2012). Public procurement design: lessons from the private sector. 
International Journal of Industrial Organization, 30, 297-302.

Tazelaar, F., \& Snijders, C. (2013). Operational risk assessments by supply chain professionals: process and performance. Journal of Operations Management, 31, 37-51.

Tomic, B., \& Milic, T. (2013). Automated interpretation of key performance indicator values and its application in education. Knowledge-Based Systems, 37, 250-260.

Turetken, O., \& Demirors, O. (2011). Plural: a decentralized business process modeling method. Information \& Management, 48, 235-247.

Walker, H., Schotanus, F., Bakker, E., \& Harland, C. (2013). Collaborative procurement: a relational view of buyer-buyer relationships. Public Administration Review, 73, 588-598.

Xia, B., Chan, A., Zuo, J., \& Molenaar, K. (2013). Analysis of selection criteria for design-builders through the analysis of requests for proposal. Journal of Management in Engineering, 29,19-24.

Xu, M., Chen, Y., \& Xu, X. (2010). The effect of demand uncertainty in a price-setting newsvendor model. European Journal of Operation Research, 207, 946-957.

Xu, M., \& Lu, Y. (2013). The effect of supply uncertainty in price-setting newsvendor model. European Journal of Operation Research, 227, 423-433.

Yu, B. T. W., To, W. M., \& Lee, P. K. C. (2012). Quality management framework for public management decision making. Management Decision, 50, 420-438.

Zhang, J., \& Chen, J. (2013). Supplier selection and procurement decisions with uncertain demand, fixed selection costs and quantity discounts. Computers \& Operations Research, 40, 2703-2710. 